Yichen Liu*, Monica Marras, and Giovanni Porru

\title{
Comparison results for nonlinear divergence structure elliptic PDE's
}

https://doi.org/10.1515/anona-2020-0008

Received September 30, 2018; accepted December 15, 2018.

Abstract: First we prove a comparison result for a nonlinear divergence structure elliptic partial differential equation. Next we find an estimate of the solution of a boundary value problem in a domain $\Omega$ in terms of the solution of a related symmetric boundary value problem in a ball $B$ having the same measure as $\Omega$. For $\mathrm{p}$ Laplace equations, the corresponding result is due to Giorgio Talenti. In a special (radial) case we also prove a reverse comparison result.

Keywords: Quasi-linear equations, Symmetrization, Comparison results

MSC: 35B51, 35J62, 49K20, 49K30

\section{Introduction}

In the seminal paper [1], Giorgio Talenti established sharp estimates of the solution to a boundary value problem of a second order elliptic partial differential equation in terms of the solution of a related symmetric problem. We refer to the survey [2] for a detailed treatment of the subject. The interest of these results relies on the obvious fact that a symmetric problem reduces to an ordinary differential equation and is easier to be solved. The papers by Talenti have inspired the use of similar methods in numerous investigations involving both linear and nonlinear elliptic problems.

To be more precise, let $\Omega \subset \mathbb{R}^{n}$ be a bounded smooth domain, let $f: \Omega \rightarrow \mathbb{R}$ be positive and bounded, and let $h: \mathbb{R}^{+} \rightarrow \mathbb{R}^{+}$be non-decreasing. Let $g$ be positive and such that $g\left(s^{2}\right) s$ is strictly increasing and differentiable for $s>0$. Let $u$ be a solution to

$$
-\left(g\left(|\nabla u|^{2}\right) u_{x_{i}}\right)_{x_{i}}=f(x) h(u), u>0 \text { in } \Omega, u=0 \text { on } \partial \Omega .
$$

Here and in what follows, the summation convention over repeated indices from 1 to $n$ is in effect. If $B \subset \mathbb{R}^{n}$ is the ball centered at the origin with the same measure as $\Omega$ and if $f^{\sharp}$ is the Schwarz (decreasing) rearrangement of $f$, let $v$ be a solution to

$$
-\left(g\left(|\nabla v|^{2}\right) v_{x_{i}}\right)_{x_{i}}=f^{\sharp}(x) h(v), \quad v>0 \text { in } B, \quad v=0 \text { on } \partial B .
$$

When

$$
u^{\sharp}(x) \leq v(x) \text { in } B \text { ? }
$$

Under suitable conditions on the function $h$, the answer is positive for the $p$-Laplacian, where $g\left(s^{2}\right)=s^{p-2}$, $p>1$. Recently, inequality (3) has been proved for the ( $p, q$ )-Laplacian, where $g\left(s^{2}\right)=s^{p-2}+s^{q-2}, p>q>1$, see [3]. In the last decades, many authors have studied $(p, q)$-Laplace equations, see [4-6] and references

*Corresponding Author: Yichen Liu, Department of Mathematical Sciences, Xi’an Jiaotong-Liverpool University, Suzhou, China, E-mail: Yichen.Liu01@xjtlu.edu.cn

Monica Marras, Department of Mathematics and Informatics, University of Cagliari, Italy, E-mail: mmarras@unica.it Giovanni Porru, Department of Mathematics and Informatics, University of Cagliari, Italy, E-mail: porru@unica.it 
therein. In this paper we show that (3) holds for a wide class of operators under appropriate conditions on $g$ and $h$.

Let us find conditions on $g$ and $h$ which ensure existence and uniqueness for problems (1) and (2). With $G(s):=g\left(s^{2}\right) s$ we assume:

(G0) There are $p \geq q>1$ and $M \geq 1$ such that

$$
\frac{1}{M} s^{p-1} \leq G(s) \leq M\left(s^{p-1}+s^{q-1}\right) \quad \forall s>0 .
$$

(G1) $G(s)$ is continuous for $s \geq 0$, is strictly increasing and continuously differentiable for $s>0$.

(G2) With $q$ as in (G0), the function

$$
\frac{G(s)}{s^{q-1}} \text { is non-decreasing for } s>0 .
$$

(H1) $h(t)$ is a positive non-decreasing function for $t>0$.

(H2) There is $1<\alpha<q$ such that $h(t) t^{1-\alpha}$ is bounded and non-increasing for $t>0$. Here $q$ is the same as in (G0).

Remark 1.1. If $1<\alpha<q$, (G2) implies

$$
\frac{G(s)}{s^{\alpha-1}} \text { is strictly increasing for } s>0 .
$$

We note that conditions (G0), (G1) and (G2) hold for a wide class of equations including the p-Laplacian and the (p,q)-Laplacian.

\section{Existence of positive solutions}

Assuming condition (G0), the natural space for solutions to problem (1) is the Sobolev space $W_{0}^{1, p}(\Omega)$. The equation in (1) is the Euler equation of the functional

$$
I(w)=\int_{\Omega}\left(\int_{0}^{|\nabla w|} G(s) d s-f(x) \int_{0}^{w} h(s) d s\right) d x .
$$

It is well-known that a function $u$ that minimizes $I(w)$ for $w \in W_{0}^{1, p}(\Omega), w \geq 0$, is a solution to the equation in (1) with $u \geq 0$. We claim that, under conditions (G1), (G2), (H1) and (H2), a minimum for $I(w)$ cannot be zero in any ball $B \subset \Omega$. Indeed, arguing by contradiction, let $\tilde{w}$ be a minimum vanishing on some ball $B$. Define $z=\tilde{w}+\epsilon \phi$, where $0<\epsilon<1$ and $\phi \in C_{0}^{1}(B)$ is a positive function in $B$ and vanishing on $\Omega \backslash B$. We have

$$
I(z)=I(\tilde{w})+\int_{B}\left(\int_{0}^{\epsilon|\nabla \phi|} G(s) d s-f(x) \int_{0}^{\epsilon \phi} h(s) d s\right) d x .
$$

We first observe that conditions (G2) and (H2) imply, for $0<\epsilon<1$ and $\tau>0$,

$$
G(\epsilon \tau) \leq \epsilon^{q-1} G(\tau), \quad h(\epsilon \tau) \geq \epsilon^{\alpha-1} h(\tau) .
$$


Putting $s=\epsilon \tau$ with $0<\epsilon<1$ and using these inequalities we find

$$
\begin{aligned}
I(z) & =I(\tilde{w})+\int_{\Omega}\left(\epsilon \int_{0}^{|\nabla \phi|} G(\epsilon \tau) d \tau-f(x) \epsilon \int_{0}^{\phi} h(\epsilon \tau) d \tau\right) d x \\
& \leq I(\tilde{w})+\int_{\Omega}\left(\epsilon^{q} \int_{0}^{|\nabla \phi|} G(\tau) d \tau-f(x) \epsilon^{\alpha} \int_{0}^{\phi} h(\tau) d \tau\right) d x \\
& =I(\tilde{w})+\epsilon^{\alpha} \int_{\Omega}\left(\epsilon^{q-\alpha} \int_{0}^{|\nabla \phi|} G(\tau) d \tau-f(x) \int_{0}^{\phi} h(\tau) d \tau\right) d x .
\end{aligned}
$$

Since $q>\alpha$, it is clear that $I(z)<I(\tilde{w})$ for $\epsilon$ small enough. The claim follows. Therefore, we may assume that there exists a solution to (1) with $u>0$ almost everywhere in $\Omega$. At the end of the next Section, we will prove that such a solution $u$ is positive in $\Omega$.

In this paper we consider solutions belonging to $C^{1}(\bar{\Omega})$. We refer to [7-10] for regularity results.

\section{A comparison result}

Lemma 3.1. Let $G(s)=g\left(s^{2}\right)$ s satisfy conditions (G1) and (G2). For $x, y \in \mathbb{R}^{n}$ and $0<t \leq 1$ we have

$$
g\left(|x|^{2}\right)\left[|x|^{2}\left(1+(\alpha-1) t^{\alpha}\right)-\alpha t^{\alpha-1} x \cdot y\right]+g\left(|y|^{2}\right)\left[|y|^{2}\left(1+(\alpha-1) t^{-\alpha}\right)-\alpha t^{-\alpha+1} x \cdot y\right] \geq 0 .
$$

In addition, if $|x|+|y|>0$ and $0<t<1$, inequality (4) holds in a strict sense. Here $1<\alpha<q$.

Proof. Recall the generalized Young's inequality

$$
x \cdot y \leq \int_{0}^{|x|} \varphi(\tau) d \tau+\int_{0}^{|y|} G(\tau) d \tau,
$$

where $\varphi(\tau)$ is the inverse function of $G(\tau)$. Replacing $x$ by $g\left(|x|^{2}\right) t^{\alpha-1} \chi$ we find

$$
g\left(|x|^{2}\right) t^{\alpha-1} x \cdot y \leq \int_{0}^{t^{\alpha-1} G(|x|)} \varphi(\tau) d \tau+\int_{0}^{|y|} G(\tau) d \tau .
$$

Similarly, we have

$$
x \cdot y \leq \int_{0}^{|y|} \varphi(\tau) d \tau+\int_{0}^{|x|} G(\tau) d \tau .
$$

Replacing $y$ by $g\left(|y|^{2}\right) t^{1-\alpha} y$ in the latter inequality we find

$$
g\left(|y|^{2}\right) t^{1-\alpha} x \cdot y \leq \int_{0}^{t^{1-\alpha} G(|y|)} \varphi(\tau) d \tau+\int_{0}^{|x|} G(\tau) d \tau .
$$

In view of (6) and (7), inequality (4) holds provided

$$
\begin{aligned}
\Psi(t):= & G(|x|)|x|\left(1+(\alpha-1) t^{\alpha}\right)-\alpha \int_{0}^{t^{\alpha-1} G(|x|)} \varphi(\tau) d \tau-\alpha \int_{0}^{|y|} G(\tau) d \tau+G(|y|)|y|\left(1+(\alpha-1) t^{-\alpha}\right) \\
& -\alpha \int_{0}^{t^{1-\alpha} G(|y|)} \varphi(\tau) d \tau-\alpha \int_{0}^{|x|} G(\tau) d \tau \geq 0 .
\end{aligned}
$$


In case of $t=1$ we have

$$
\begin{aligned}
& \Psi(1)=\alpha\left[G(|x|)|x|-\int_{0}^{G(|x|)} \varphi(\tau) d \tau-\int_{0}^{|y|} G(\tau) d \tau\right. \\
& \left.+G(|y|)|y|-\int_{0}^{G(|y|)} \varphi(\tau) d \tau-\int_{0}^{|x|} G(\tau) d \tau\right] .
\end{aligned}
$$

Putting $\tau=G(s)$ we find

$$
\int_{0}^{G(|x|)} \varphi(\tau) d \tau=\int_{0}^{|x|} s G^{\prime}(s) d s=|x| G(|x|)-\int_{0}^{|x|} G(s) d s .
$$

Similarly, we find

$$
\int_{0}^{G(|y|)} \varphi(\tau) d \tau=|y| G(|y|)-\int_{0}^{|y|} G(s) d s .
$$

Insertion of (10) and (11) into (9) yields $\Psi(1)=0$. Hence, to prove that $\Psi(t)>0$ for $0<t<1$ when $|x|+|y|>0$, it is enough to prove that $\Psi^{\prime}(t)<0$. Since

$$
\Psi^{\prime}(t)=\frac{\alpha(\alpha-1)}{t}\left[G(|x|)|x| t^{\alpha}-\varphi\left(t^{\alpha-1} G(|x|)\right) t^{\alpha-1} G(|x|)-G(|y|)|y| t^{-\alpha}+\varphi\left(t^{1-\alpha} G(|y|)\right) t^{1-\alpha} G(|y|)\right]
$$

we must show that

$$
G(|x|)|x| t^{\alpha}-\varphi\left(t^{\alpha-1} G(|x|)\right) t^{\alpha-1} G(|x|)<G(|y|)|y| t^{-\alpha}-\varphi\left(t^{1-\alpha} G(|y|)\right) t^{1-\alpha} G(|y|)
$$

Let us prove that the left hand side of (12) is negative when $|x|>0$. Indeed, if $|x|>0$, the inequality

$$
G(|x|)|x| t^{\alpha}-\varphi\left(t^{\alpha-1} G(|x|)\right) t^{\alpha-1} G(|x|)<0
$$

is equivalent to

$$
t|x|<\varphi\left(t^{\alpha-1} G(|x|)\right),
$$

which in turn is equivalent to

$$
G(t|x|)<t^{\alpha-1} G(|x|) .
$$

The latter inequality can be rewritten as

$$
\frac{G(t|x|)}{(t|x|)^{\alpha-1}}<\frac{G(|x|)}{|x|^{\alpha-1}}
$$

which holds by Remark 1.1.

Now, let us prove that the right hand side of (12) is positive when $|y|>0$, that is

$$
G(|y|)|y| t^{-\alpha}-\varphi\left(t^{1-\alpha} G(|y|)\right) t^{1-\alpha} G(|y|)>0 .
$$

Let us write this inequality as

$$
t^{-1}|y|>\varphi\left(t^{1-\alpha} G(|y|)\right)
$$

which can be rewritten as

$$
G\left(t^{-1}|y|\right)>t^{1-\alpha} G(|y|)
$$

This inequality is equivalent to the following

$$
\frac{G(|y|)}{|y|^{\alpha-1}}<\frac{G\left(t^{-1}|y|\right)}{\left(t^{-1}|y|\right)^{\alpha-1}},
$$

which holds by Remark 1.1. Hence, inequality (12) holds when $|x|+|y|>0$. It follows that also (8) and (4) hold in a strict sense for $0<t<1$. The lemma is proved. 
Theorem 3.2. Let $G(s):=g\left(s^{2}\right)$ s satisfy $(G 0),(G 1)$ and $(G 2)$, and let $h(t)$ satisfy $(H 1)$ and $(H 2)$. Let $u \in C^{1}(\bar{\Omega})$, $u=0$ on $\partial \Omega$ and $u>0$ everywhere on $\Omega$ such that

$$
\int_{\Omega} g\left(|\nabla u|^{2}\right) \nabla u \cdot \nabla \phi d x \leq \int_{\Omega} f(x) h(u) \phi d x \forall \phi \in C_{0}^{1}(\Omega), \quad \phi \geq 0 .
$$

Let $v \in C^{1}(\bar{\Omega}), v \geq 0$ on $\partial \Omega$ and $v>0$ everywhere on $\Omega$ such that

$$
\int_{\Omega} g\left(|\nabla v|^{2}\right) \nabla v \cdot \nabla \phi d x \geq \int_{\Omega} f(x) h(v) \phi d x \forall \phi \in C_{0}^{1}(\Omega), \quad \phi \geq 0 .
$$

Then $u \leq v$ in $\Omega$.

Proof. Define $A=\{x \in \Omega: u(x)>v(x)\}$. If we prove that $A$ is empty, the assertion of the theorem follows. We argue by contradiction, assuming $A$ is not empty. For $\epsilon>0$, define $u_{\epsilon}=u+\epsilon$ and $v_{\epsilon}=v+\epsilon$. Note that we have $u_{\epsilon}(x)>v_{\epsilon}(x)$ in $A$. Using

$$
\phi_{1}(x)=\max \left[\frac{u_{\epsilon}^{\alpha}(x)-v_{\epsilon}^{\alpha}(x)}{u_{\epsilon}^{\alpha-1}(x)}, 0\right]
$$

as test function in (13) we obtain

$$
\int_{A} g\left(|\nabla u|^{2}\right) \nabla u \cdot \nabla\left(\frac{u_{\epsilon}^{\alpha}-v_{\epsilon}^{\alpha}}{u_{\epsilon}^{\alpha-1}}\right) d x \leq \int_{A} f(x) \frac{h(u)}{u^{\alpha-1}}\left(u_{\epsilon}^{\alpha}-v_{\epsilon}^{\alpha}\right)\left(\frac{u}{u_{\epsilon}}\right)^{\alpha-1} d x .
$$

Similarly, using

$$
\phi_{2}(x)=\max \left[\frac{u_{\epsilon}^{\alpha}(x)-v_{\epsilon}^{\alpha}(x)}{v_{\epsilon}^{\alpha-1}(x)}, 0\right]
$$

as test function in (14) we obtain

$$
\int_{A} g\left(|\nabla v|^{2}\right) \nabla v \cdot \nabla\left(\frac{u_{\epsilon}^{\alpha}-v_{\epsilon}^{\alpha}}{v_{\epsilon}^{\alpha-1}}\right) d x \geq \int_{A} f(x) \frac{h(v)}{v^{\alpha-1}}\left(u_{\epsilon}^{\alpha}-v_{\epsilon}^{\alpha}\right)\left(\frac{v}{v_{\epsilon}}\right)^{\alpha-1} d x .
$$

Subtracting the latter inequality from the previous one we get

$$
\begin{aligned}
& \int_{A}\left[g\left(|\nabla u|^{2}\right) \nabla u \cdot \nabla\left(\frac{u_{\epsilon}^{\alpha}-v_{\epsilon}^{\alpha}}{u_{\epsilon}^{\alpha-1}}\right)+g\left(|\nabla v|^{2}\right) \nabla v \cdot \nabla\left(\frac{v_{\epsilon}^{\alpha}-u_{\epsilon}^{\alpha}}{v_{\epsilon}^{\alpha-1}}\right)\right] d x \\
& \leq \int_{A} f(x)\left[\frac{h(u)}{u^{\alpha-1}}\left(\frac{u}{u_{\epsilon}}\right)^{\alpha-1}-\frac{h(v)}{v^{\alpha-1}}\left(\frac{v}{v_{\epsilon}}\right)^{\alpha-1}\right]\left(u_{\epsilon}^{\alpha}-v_{\epsilon}^{\alpha}\right) d x .
\end{aligned}
$$

Since

$$
\nabla\left(\frac{u_{\epsilon}^{\alpha}-v_{\epsilon}^{\alpha}}{u_{\epsilon}^{\alpha-1}}\right)=\nabla u+(\alpha-1)\left(\frac{v_{\epsilon}}{u_{\epsilon}}\right)^{\alpha} \nabla u-\alpha\left(\frac{v_{\epsilon}}{u_{\epsilon}}\right)^{\alpha-1} \nabla v
$$

and

$$
\nabla\left(\frac{v_{\epsilon}^{\alpha}-u_{\epsilon}^{\alpha}}{v_{\epsilon}^{\alpha-1}}\right)=\nabla v+(\alpha-1)\left(\frac{u_{\epsilon}}{v_{\epsilon}}\right)^{\alpha} \nabla v-\alpha\left(\frac{u_{\epsilon}}{v_{\epsilon}}\right)^{\alpha-1} \nabla u,
$$

we find

$$
\begin{aligned}
& \int_{A}\left[g\left(|\nabla u|^{2}\right) \nabla u \cdot \nabla\left(\frac{u_{\epsilon}^{\alpha}-v_{\epsilon}^{\alpha}}{u_{\epsilon}^{\alpha-1}}\right)+g\left(|\nabla v|^{2}\right) \nabla v \cdot \nabla\left(\frac{v_{\epsilon}^{\alpha}-u_{\epsilon}^{\alpha}}{v_{\epsilon}^{\alpha-1}}\right)\right] d x \\
& =\int_{A}\left\{g\left(|\nabla u|^{2}\right)\left[|\nabla u|^{2}\left(1+(\alpha-1)\left(\frac{v_{\epsilon}}{u_{\epsilon}}\right)^{\alpha}\right)-\alpha\left(\frac{v_{\epsilon}}{u_{\epsilon}}\right)^{\alpha-1} \nabla u \cdot \nabla v\right]\right. \\
& \left.+g\left(|\nabla v|^{2}\right)\left[|\nabla v|^{2}\left(1+(\alpha-1)\left(\frac{u_{\epsilon}}{v_{\epsilon}}\right)^{\alpha}\right)-\alpha\left(\frac{u_{\epsilon}}{v_{\epsilon}}\right)^{\alpha-1} \nabla v \cdot \nabla u\right]\right\} d x .
\end{aligned}
$$


By Lemma 3.1 with $x=\nabla u, y=\nabla v$ and $t=\frac{v_{\epsilon}}{u_{\epsilon}}$ we have

$$
\begin{aligned}
& g\left(|\nabla u|^{2}\right)\left[|\nabla u|^{2}\left(1+(\alpha-1)\left(\frac{v_{\epsilon}}{u_{\epsilon}}\right)^{\alpha}\right)-\alpha\left(\frac{v_{\epsilon}}{u_{\epsilon}}\right)^{\alpha-1} \nabla u \cdot \nabla v\right] \\
& +g\left(|\nabla v|^{2}\right)\left[|\nabla v|^{2}\left(1+(\alpha-1)\left(\frac{u_{\epsilon}}{v_{\epsilon}}\right)^{\alpha}\right)-\alpha\left(\frac{u_{\epsilon}}{v_{\epsilon}}\right)^{\alpha-1} \nabla v \cdot \nabla u\right] \geq 0 .
\end{aligned}
$$

Therefore, using Fatou's Lemma we find

$$
\begin{aligned}
& \liminf _{\epsilon \rightarrow 0} \int_{A}\left[g\left(|\nabla u|^{2}\right) \nabla u \cdot \nabla\left(\frac{u_{\epsilon}^{\alpha}-v_{\epsilon}^{\alpha}}{u_{\epsilon}^{\alpha-1}}\right)+g\left(|\nabla v|^{2}\right) \nabla v \cdot \nabla\left(\frac{v_{\epsilon}^{\alpha}-u_{\epsilon}^{\alpha}}{v_{\epsilon}^{\alpha-1}}\right)\right] d x \\
& \geq \int_{A}\left\{g\left(|\nabla u|^{2}\right)\left[|\nabla u|^{2}\left(1+(\alpha-1)\left(\frac{v}{u}\right)^{\alpha}\right)-\alpha\left(\frac{v}{u}\right)^{\alpha-1} \nabla u \cdot \nabla v\right]\right. \\
& \left.+g\left(|\nabla v|^{2}\right)\left[|\nabla v|^{2}\left(1+(\alpha-1)\left(\frac{u}{v}\right)^{\alpha}\right)-\alpha\left(\frac{u}{v}\right)^{\alpha-1} \nabla v \cdot \nabla u\right]\right\} d x .
\end{aligned}
$$

On the other hand, using conditions (H1) and (H2) and Lebesgue dominated theorem we find

$$
\lim _{\epsilon \rightarrow 0} \int_{A} f(x)\left[\frac{h(u)}{u^{\alpha-1}}\left(\frac{u}{u_{\epsilon}}\right)^{\alpha-1}-\frac{h(v)}{v^{\alpha-1}}\left(\frac{v}{v_{\epsilon}}\right)^{\alpha-1}\right]\left(u_{\epsilon}^{\alpha}-v_{\epsilon}^{\alpha}\right) d x=\int_{A} f(x)\left[\frac{h(u)}{u^{\alpha-1}}-\frac{h(v)}{v^{\alpha-1}}\right]\left(u^{\alpha}-v^{\alpha}\right) d x \leq 0
$$

In view of the latter inequality and (17), from (15) as $\epsilon \rightarrow 0$ we find

$$
\begin{aligned}
& \int_{A}\left\{g\left(|\nabla u|^{2}\right)\left[|\nabla u|^{2}\left(1+(\alpha-1)\left(\frac{v}{u}\right)^{\alpha}\right)-\alpha\left(\frac{v}{u}\right)^{\alpha-1} \nabla u \cdot \nabla v\right]\right. \\
& \left.+g\left(|\nabla v|^{2}\right)\left[|\nabla v|^{2}\left(1+(\alpha-1)\left(\frac{u}{v}\right)^{\alpha}\right)-\alpha\left(\frac{u}{v}\right)^{\alpha-1} \nabla v \cdot \nabla u\right]\right\} d x \leq 0 .
\end{aligned}
$$

By (16), (18) and Lemma 3.1, we must have $|\nabla u|=|\nabla v|=0$ in $A$. Therefore, $\nabla(u-v)=0$ in $A$ and $u-v=0$ on $\partial A$. Then, $u(x)=v(x)$, contradicting the definition of $A$. The theorem follows.

As an application of Theorem 3.2, we can show that problem (1) has a (positive) solution. Indeed, we know that there is a solution $u$ such that $u>0$ almost everywhere in $\Omega$. Let $x \in \Omega$ and let $B$ be a ball centered at $x$ and contained in $\Omega$. The function $u$ satisfies

$$
-\operatorname{div}\left(g\left(|\nabla u|^{2}\right) \nabla u\right) \geq \underline{f} h(u) \text { in } B, u \geq 0 \text { on } \partial B,
$$

where $f$ is the inferior of $f$ in $B$. Now, consider a radially symmetric function $z$ such that

$$
-\operatorname{div}\left(g\left(|\nabla z|^{2}\right) \nabla z\right)=\underline{f} h(z) \text { in } B, \quad z=0 \text { on } \partial B .
$$

The function $z$ satisfies (see the last section of the present paper)

$$
-r^{n-1} g\left(\left(z^{\prime}\right)^{2}\right) z^{\prime}=\underset{-}{f} \int_{0}^{r} s^{n-1} h(z(s)) d s .
$$

Here $r=|y-x|$ for $y \in B$. It follows that $z^{\prime}(r)<0$ and $z(x)>0$. Now we apply Theorem 3.2 with $\Omega=B, f=\underline{f}$, $u=z$ and $v=u$. We find $0<z(x) \leq u(x)$. Since $x$ is arbitrary, we have $u(x)>0$ in $\Omega$.

Corollary 3.1. Let $G(s):=g\left(s^{2}\right) s$ satisfy (GO), (G1) and (G2), and let $h(t)$ satisfy (H1) and (H2). Problems (1) and (2) have a unique solution. 


\section{Extension of a Talenti's result}

In what follows we shall use the Hardy-Littlewood inequalities, namely

$$
\int_{\Omega} f(x) g(x) d x \leq \int_{0}^{|\Omega|} f^{\star}(\tau) g^{*}(\tau) d \tau,
$$

and

$$
\int_{\Omega} f(x) g(x) d x \geq \int_{0}^{|\Omega|} f_{\star}(\tau) g^{\star}(\tau) d \tau,
$$

where $f$ and $g$ are non-negative bounded functions, $f^{\star}$ and $f_{\star}$ are the decreasing and, respectively, the increasing rearrangement of $f$, see [11].

We also use the Jensen inequality, that is

$$
J\left(\frac{1}{|\Sigma|} \int_{\Sigma} f(\sigma) d \sigma\right) \leq \frac{1}{|\Sigma|} \int_{\Sigma} J(f(\sigma)) d \sigma,
$$

where the function $J$ is positive and convex, and $f$ is non-negative and integrable in $\Sigma$.

To prove our next result we need a further condition on $G$, namely

(G3) There are $y \geq 1$ and $L>0$ such that

$$
J(s):=G\left(s^{y}\right) \text { is convex for } 0<s<L .
$$

Theorem 4.1. Let $G(s):=g\left(s^{2}\right) s$ satisfy (G0), (G1), (G2) and (G3), and let $h(t)$ satisfy (H1) and (H2). If $u \in$ $W_{0}^{1, p}(\Omega) \cap C^{1}(\bar{\Omega})$ and $v \in W_{0}^{1, p}(\Omega) \cap C^{1}(\bar{\Omega})$ are solutions to (1) and (2) respectively then we have $u^{\sharp}(x) \leq v(x)$ in $\Omega^{\sharp}$.

Proof. For $t \geq 0$, let $\Omega(t)=\{x \in \Omega: u(x)>t\}$ and $\mu(t)=|\Omega(t)|$. If we set $\Sigma(t)=\{x \in \Omega: u(x)=t\}$ and integrate the equation in (1) over $\Omega(t)$ we find

$$
\int_{\Sigma(t)} G(|\nabla u|) d \sigma=\int_{\Omega(t)} f(x) h(u(x)) d x \leq \int_{0}^{\mu(t)} f^{\star}(\tau) h\left(u^{\star}(\tau)\right) d \tau .
$$

Take $L$ large enough such that $|\nabla u| \leq L$ in $\Omega$ and $|\nabla v| \leq L$ in $B$, and let $y$ as in condition (G3). Inequality (19) with $J(s)=G\left(s^{y}\right)$ and $f=|\nabla u|^{\frac{1}{y}}$ yields

$$
G\left(\left(\frac{1}{|\Sigma(t)|} \int_{\Sigma(t)}|\nabla u|^{\frac{1}{y}} d \sigma\right)^{y}\right) \leq \frac{1}{|\Sigma(t)|} \int_{\Sigma(t)} G\left(\left(|\nabla u|^{\frac{1}{y}}\right)^{y}\right) d \sigma=\frac{1}{|\Sigma(t)|} \int_{\Sigma(t)} G(|\nabla u|) d \sigma .
$$

With $r-1=1 / y$ we find

$$
G\left(\left(\frac{1}{|\Sigma(t)|} \int_{\Sigma(t)}|\nabla u|^{r-1} d \sigma\right)^{\frac{1}{r-1}}\right) \leq \frac{1}{|\Sigma(t)|} \int_{\Sigma(t)} G(|\nabla u|) d \sigma .
$$

Now, by Hölder inequality we have

$$
|\Sigma(t)|=\int_{\Sigma(t)}|\nabla u|^{-\frac{r-1}{r}}|\nabla u|^{\frac{r-1}{r}} d \sigma \leq\left(\int_{\Sigma(t)}|\nabla u|^{-1} d \sigma\right)^{\frac{r-1}{r}}\left(\int_{\Sigma(t)}|\nabla u|^{r-1} d \sigma\right)^{\frac{1}{r}},
$$

from which we find

$$
|\Sigma(t)|^{r-1} \leq\left(\int_{\Sigma(t)}|\nabla u|^{-1} d \sigma\right)^{r-1} \frac{1}{|\Sigma(t)|} \int_{\Sigma(t)}|\nabla u|^{r-1} d \sigma .
$$


On the other hand, using the Federer co-area formula

$$
-\mu^{\prime}(t)=\int_{\Sigma(t)}|\nabla u|^{-1} d \sigma
$$

and the isoperimetric inequality

$$
C_{n}(\mu(t))^{\frac{n-1}{n}} \leq|\Sigma(t)|, \quad C_{n}=n \omega_{n}^{\frac{1}{n}},
$$

inequality (23) yields

$$
\left(\frac{C_{n}(\mu(t))^{\frac{n-1}{n}}}{-\mu^{\prime}(t)}\right)^{r-1} \leq \frac{1}{|\Sigma(t)|} \int_{\Sigma(t)}|\nabla u|^{r-1} d \sigma
$$

from which we find

$$
G\left(\frac{C_{n}(\mu(t))^{\frac{n-1}{n}}}{-\mu^{\prime}(t)}\right) \leq G\left(\left(\frac{1}{|\Sigma(t)|} \int_{\Sigma(t)}|\nabla u|^{r-1} d \sigma\right)^{\frac{1}{r-1}}\right) .
$$

As usual, we denote by $\omega_{n}$ the measure of the unit ball in $\mathbb{R}^{n}$. Inserting the previous estimate into (22) we find

$$
G\left(\frac{C_{n}(\mu(t))^{\frac{n-1}{n}}}{-\mu^{\prime}(t)}\right) \leq \frac{1}{|\Sigma(t)|} \int_{\Sigma(t)} G(|\nabla u|) d \sigma
$$

Using again the isoperimetric inequality, the latter inequality yields

$$
C_{n}(\mu(t))^{\frac{n-1}{n}} G\left(\frac{C_{n}(\mu(t))^{\frac{n-1}{n}}}{-\mu^{\prime}(t)}\right) \leq \int_{\Sigma(t)} G(|\nabla u|) d \sigma .
$$

Inequalities (24) and (20) yield

$$
C_{n}(\mu(t))^{\frac{n-1}{n}} G\left(\frac{C_{n}(\mu(t))^{\frac{n-1}{n}}}{-\mu^{\prime}(t)}\right) \leq \int_{0}^{\mu(t)} f^{\star}(\tau) h\left(u^{\star}(\tau)\right) d \tau .
$$

Putting $\mu(t)=s$ and recalling that $\mu(t)$ is essentially the inverse function of $u^{*}(s)$, the latter inequality reads as

$$
C_{n} s^{\frac{n-1}{n}} G\left(C_{n} s^{\frac{n-1}{n}}\left(-\frac{d u^{\star}}{d s}\right)\right) \leq \int_{0}^{s} f^{\star}(\tau) h\left(u^{\star}(\tau)\right) d \tau .
$$

Now, let

$$
-\left(g\left(|\nabla z|^{2}\right) z_{x_{i}}\right)_{x_{i}}=f^{\sharp}(x) h\left(u^{\sharp}\right) \text { in } B, \quad z=0 \text { on } \partial B .
$$

Since the domain $B$ and the function $f^{\sharp}(x) h\left(u^{\sharp}(x)\right)$ are radially symmetric, problem (26) as a unique solution $z(x)$ which is radially symmetric. As a consequence, all level sets of $z(x)$ are balls. Arguing as in the previous case and observing that the inequalities are now equalities, instead of (25) we find

$$
C_{n} s^{\frac{n-1}{n}} G\left(C_{n} s^{\frac{n-1}{n}}\left(-\frac{d z^{\star}}{d s}\right)\right)=\int_{0}^{s} f^{\star}(\tau) h\left(u^{\star}(\tau)\right) d \tau .
$$

From (25) and (27) it follows that

$$
C_{n} s^{\frac{n-1}{n}} G\left(C_{n} s^{\frac{n-1}{n}}\left(-\frac{d u^{\star}}{d s}\right)\right) \leq C_{n} s^{\frac{n-1}{n}} G\left(C_{n} s^{\frac{n-1}{n}}\left(-\frac{d z^{\star}}{d s}\right)\right) .
$$

Since $G$ is strictly increasing, we find

$$
C_{n} s^{\frac{n-1}{n}}\left(-\frac{d u^{\star}}{d s}\right) \leq C_{n} s^{\frac{n-1}{n}}\left(-\frac{d z^{\star}}{d s}\right)
$$


whence

$$
-\frac{d u^{\star}}{d s} \leq-\frac{d z^{\star}}{d s}
$$

Integrating over $(s,|B|)$ and recalling that $u^{*}(|B|)=z^{\star}(|B|)=0$ we find

$$
u^{\star}(s) \leq z^{\star}(s), \quad 0<s<|B|,
$$

and

$$
u^{\sharp}(x) \leq z(x), \quad \forall x \in B .
$$

Insertion of (29) into (26) yields

$$
-\left(g\left(|\nabla z|^{2}\right) z_{x_{i}}\right)_{x_{i}} \leq f^{\sharp}(x) h(z) \text { in } B, z=0 \text { on } \partial B .
$$

Theorem 3.2 applied to (2) and (30) yields

$$
z(x) \leq v(x) .
$$

The latter inequality and (29) yield the desired result. The theorem is proved.

\section{The radial case}

In this section we consider the case $\Omega$ is a ball $B \subset \mathbb{R}^{n}$ centered at the origin and radius $R$, and $f(x)$ is a positive radial function. We write $f(x)=f(r)$ with $|x|=r$. Of course, Theorem 4.1 continuous to hold. Here we prove a reverse comparison result involving the increasing rearrangement of $f$.

Let $h: \mathbb{R}^{+} \rightarrow \mathbb{R}^{+}$be non-decreasing, and let $g$ be positive and such that $g\left(s^{2}\right) s$ is strictly increasing for $s>0$. Consider the boundary value problems

$$
\begin{gathered}
-\left(g\left(|\nabla v|^{2}\right) v_{x_{i}}\right)_{x_{i}}=f(x) h(v) \text { in } B, \quad v=0 \text { on } \partial B, \\
-\left(g\left(|\nabla w|^{2}\right) w_{x_{i}}\right)_{x_{i}}=f_{\sharp}(x) h(w) \text { in } B, w=0 \text { on } \partial B .
\end{gathered}
$$

Here $f_{\sharp}$ is the Schwarz increasing rearrangement of $f$, that is, $f_{\sharp}(x)=f_{\star}\left(\omega_{n}|x|^{n}\right), f_{\star}(s)=f^{\star}(|B|-s)$.

Theorem 5.1. Let $G(s):=g\left(s^{2}\right)$ s satisfy $(G 0),(G 1)$ and $(G 2)$, and let $h(t)$ satisfy $(H 1)$ and $(H 2)$. If $v \in W_{0}^{1, p}(\Omega) \cap$ $C^{1}(\bar{\Omega})$ and $w \in W_{0}^{1, p}(\Omega) \cap C^{1}(\bar{\Omega})$ are solutions to (31) and (32) respectively then we have $v(x) \geq w(x)$ in $B$.

Proof. By Theorem 3.2, problems (31) and (32) have a unique positive solution $v(x)$ and $w(x)$ respectively. Hence, since $B$ is radially symmetric, $v(x)$ and $w(x)$ must be radially symmetric. The equation in (31) can be rewritten as

$$
-g\left(|\nabla v|^{2}\right) \Delta v-2 g^{\prime} v^{\prime \prime}\left(v^{\prime}\right)^{2}=f(r) h(v) .
$$

Recall what we are writing $f(r)=f(x)$ with $r=|x|$. We find

$$
-r^{n-1} g v^{\prime \prime}-(n-1) r^{n-2} g v^{\prime}-2 r^{n-1} g^{\prime} v^{\prime \prime}\left(v^{\prime}\right)^{2}=r^{n-1} f(r) h(v),
$$

which can be written as

$$
-\left(r^{n-1} g v^{\prime}\right)^{\prime}=r^{n-1} f(r) h(v)
$$

Integration over $(0, r)$ yields

$$
-r^{n-1} g v^{\prime}=\int_{0}^{r} \rho^{n-1} f(\rho) h(v(\rho)) d \rho=\frac{1}{n \omega_{n}} \int_{B(r)} f(x) h(v(x)) d x,
$$


where $B(r)$ is the ball concentric with $B$ and radius $r$. By (33) we have $v^{\prime}(r)<0$ for $0<r<R$. Since $v(R)=0$, $v(r)$ is positive and decreasing. Moreover, (33) and the Hardy-Littlewood inequality

$$
\int_{B(r)} f(x) h(v) d x \geq \int_{B(r)} f_{\sharp}(x) h(v) d x
$$

yield

$$
-r^{n-1} g v^{\prime} \geq \frac{1}{n \omega_{n}} \int_{B(r)} f_{\sharp}(x) h(v) d x .
$$

Now, let

$$
-\left(g\left(|\nabla z|^{2}\right) z_{x_{i}}\right)_{x_{i}}=f_{\sharp}(x) h(v) \text { in } B, z=0 \text { on } \partial B .
$$

The solution $z$ is a radial function. Arguing as in the previous case, one finds

$$
-r^{n-1} g z^{\prime}=\frac{1}{n \omega_{n}} \int_{B(r)} f_{\sharp}(x) h(v) d x .
$$

Inequality (34) and the latter equation yield

$$
r^{n-1} g\left(\left(v^{\prime}\right)^{2}\right)\left(-v^{\prime}\right) \geq r^{n-1} g\left(\left(z^{\prime}\right)^{2}\right)\left(-z^{\prime}\right) .
$$

Since $g\left(s^{2}\right) s$ is strictly increasing, it follows that

$$
-v^{\prime} \geq-z^{\prime}
$$

Integrating over $(r, R)$ and recalling that $z(R)=v(R)=0$ we find $v(r) \geq z(r)$ for $0<r<R$, and

$$
v(x) \geq z(x), \quad \forall x \in B .
$$

Using the latter inequality and (35) we get

$$
-\left(g\left(|\nabla z|^{2}\right) z_{x_{i}}\right)_{x_{i}} \geq f_{\sharp}(x) h(z) \text { in } B, \quad z=0 \text { on } \partial B .
$$

Applying Theorem 3.2 to problems (37) and (32) we find

$$
z(x) \geq w(x) .
$$

The latter inequality and (36) yield the desired result. The theorem is proved.

Acknowledgement. This work was initiated when Y. Liu visited the University of Cagliari, Italy. He would like to thank Professors M. Marras, G. Porru and S. Vernier-Piro for their hospitality. Y. Liu is supported by the XJTLU's research fund RDF-17-02-22.

\section{References}

[1] G. Talenti, Elliptic equations and rearrangements, Ann. Scuola Norm. Sup. Pisa Cl. Sci. 3, (1976), no. 4, 697-718.

[2] G. Talenti, The art of rearranging, Milan J. Math. 84, (2016), no. 1, 105-157.

[3] B. Emamizadeh, Y. Liu and G. Porru, Extensions of a result by G. Talenti to (p,q)-Laplace equations, Nonlinear Anal. 180, (2019), 41-51.

[4] P. Candito, S.A. Marano and K. Perera, On a class of critical (p,q)-Laplacian problems, NoDEA Nonlinear Differential Equations Appl. 22, (2015), no. 6, 1959-1972.

[5] S. A. Marano and N. S. Papageorgiou, Constant-sign and nodal solutions of coercive (p,q)-Laplacian problems, Nonlinear Anal. 77, (2013), 118-129.

[6] M. Tanaka, Uniqueness of a positive solution and existence of a sign-changing solution for (p,q)-Laplace equations, J. Nonlinear Funct. Anal. 2014, (2014), Article ID 14. 
[7] D. Gilbarg and N.S. Trudinger, Elliptic Partial Differential Equations of Second Order, Spring-Verlag, New York-BerlinHeidelberg, 1977.

[8] G. M. Lieberman, Boundary regularity for solutions of degenerate elliptic equations, Nonlinear Anal. 12, (1988), no. 11, 1203-1219.

[9] G. M. Lieberman, The natural generalization of the natural conditions of Ladyzhenskaya and Ural'tseva for elliptic equations, Comm. Partial Differential Equations 16, (1991), no. 2-3, 311-361.

[10] P. Pucci and J. Serrin, The maximum principle, Birkhäuser Verlag, Basel, 2007.

[11] B. Kawohl, Rearrangements and Convexity of level sets in PDE's, Lectures Notes in Mathematics 1150, Springer-Verlag, Berlin, 1985. 